\title{
Local Segregation Scores of Entropy-Based Segregation Indices
}

\author{
Benjamin Elbers \\ be2239@columbia.edu \\ Department of Sociology, Columbia University
}

This version: March 8, 2021

\begin{abstract}
The Mutual Information segregation index $M$ can be decomposed into a weighted average of local segregation scores. This useful property can be used to assess whether some units (say, occupations or geographic areas) contribute more to overall segregation than other units. The related segregation index $H$ is a normalized version of the $M$ index, such that the index is constrained to fall between 0 and 1 . The question addressed in this paper is whether local segregation scores of the $M$ index can be normalized in a similar way, to arrive at useful local segregation scores for the $H$ index. The paper shows that it is not possible to obtain normalized local segregation scores that fall between 0 and 1 and that also aggregate to the $H$ index. The one exception to this is the situation when all groups in the population are exactly of equal size. It is also (trivially) possible to decompose the $H$ index into weighted local segregation scores, however, they have the same problems of interpretation as the local segregation scores of the $M$ index.
\end{abstract}

\section{Notation}

To define the $M$ index (Theil, 1972; Mora and Ruiz-Castillo, 2011; Elbers, 2021), let $t_{u g}$ be the entries of a matrix with $U$ rows (say, occupations or spatial units) and $G$ columns (say, racial groups or genders). Let $t$ be the total population of the matrix, $t=\sum_{u=1}^{U} \sum_{g=1}^{G} t_{u g}$. The joint probability of being in unit $u$ and group $g$ is $p_{u g}=t_{u g} / t$. Also define $p_{u}$. $=\sum_{g=1}^{G} t_{u g} / t$ and $p_{\cdot g}=\sum_{u=1}^{U} t_{u g} / t$ as the marginal probabilities of spatial units and racial groups, respectively. The index $M$ is then defined as

$$
M=\sum_{u=1}^{U} \sum_{g=1}^{G} p_{u g} \log \left(\frac{p_{u g}}{p_{u} \cdot p_{\cdot g}}\right),
$$

where here (and throughout the paper), define $\log 0 \equiv 0$. Note that this "index" is exactly identical to the concept of mutual information from information theory.

Alternatively, we can derive the $M$ index from local segregation scores. Let $p_{g \mid u}=t_{u g} / t_{u}$. be the proportion of group $g$ within unit $u$. Then the local segregation score for unit $u$ is

$$
L_{u}=\sum_{g=1}^{G} p_{g \mid u} \log \left(\frac{p_{g \mid u}}{p_{\cdot g}}\right) .
$$


These local segregation scores are now related to the $M$ index by the following simple identity:

$$
M=\sum_{u=1}^{U} p_{u} \cdot L_{u} .
$$

Hence, the overall $M$ index is simply a weighted average of the local segregation scores.

To define the $H$ index, let $E(\mathbf{p})=-\sum p_{i} \log p_{i}$ denote the entropy of the vector $\mathbf{p}$, where the summation extends over the elements of $\mathbf{p}$, and $\mathbf{p}$ forms a valid probability distribution (in the sense that $\sum p_{i}=1$ and $p_{i} \geq 0$ for all $i$ ). As useful notation, we will write $\mathbf{p}_{\cdot g}=$ $\left(p_{\cdot 1}, p_{\cdot 2}, \ldots, p_{\cdot G}\right)$ to collect the elements of the relevant distribution in a vector. The $H$ index is a normalized version of the $M$ index such that the index ranges from 0 to 1 . It is defined by dividing the $M$ index by the marginal group entropy, $E\left(\mathbf{p}_{. g}\right)$ :

$$
H=\frac{M}{E\left(\mathbf{p}_{\cdot g}\right)} .
$$

This normalization is very useful for interpretation, and this index has been extensively used in the segregation literature as the "Theil index" (e.g., Reardon and Firebaugh, 2002) The question remains whether there is a similar, useful normalization for local segregation scores as in (1).

\section{Properties of local segregation scores}

To study the properties of local segregation scores, it is useful to define the following three information-theoretic quantities. ${ }^{1}$ Let $\mathbf{p}$ and $\mathbf{q}$ denote vectors of identical size, where each vector forms a probability distribution. Then

$$
\begin{aligned}
D_{K L}(\mathbf{p} \| \mathbf{q}) & =\sum p_{i} \log \left(\frac{p_{i}}{q_{i}}\right), \\
E(\mathbf{p}) & =-\sum p_{i} \log \left(p_{i}\right), \\
E(\mathbf{p}, \mathbf{q}) & =-\sum p_{i} \log \left(q_{i}\right),
\end{aligned}
$$

where $D_{K L}(\mathbf{p} \| \mathbf{q})$ is the Kullback-Leibler divergence of distributions $\mathbf{p}$ and $\mathbf{q}, E(\mathbf{p})$ is the entropy of distribution $\mathbf{p}$, and $E(\mathbf{p}, \mathbf{q})$ is the cross-entropy of distributions $\mathbf{p}$ and $\mathbf{q}$. Some algebra shows that the following identity holds:

$$
E(\mathbf{p}, \mathbf{q})=E(\mathbf{p})+D_{K L}(\mathbf{p} \| \mathbf{q})
$$

This relationship will be useful, as the local segregation score $L_{u}$ in (1) is simply the KullbackLeibler divergence of distributions $\mathbf{p}_{g \mid u}$ and $\mathbf{p} \cdot g$. Hence,

$$
\begin{aligned}
L_{u} & =D_{K L}\left(\mathbf{p}_{g \mid u} \| \mathbf{p}_{\cdot g}\right) \\
& =E\left(\mathbf{p}_{g \mid u}, \mathbf{p}_{g}\right)-E\left(\mathbf{p}_{g \mid u}\right) .
\end{aligned}
$$

\footnotetext{
${ }^{1}$ In information theory, the entropy and cross-entropy are usually denoted by $H(\cdot)$ and $H(\cdot, \cdot)$. In the segregation literature, unfortunately, the symbol $H$ was chosen to denote the normalized mutual information index.
} 
This identity can be used to derive minimum and maximum values for $L_{u}$. First, if a unit is minimally segregated, the unit-specific group distribution $\mathbf{p}_{g \mid u}$ is identical to the overall group distribution $\mathbf{p}_{\cdot g}$. If this is the case, $E\left(\mathbf{p}_{g \mid u}, \mathbf{p}_{\cdot g}\right)=E\left(\mathbf{p}_{g \mid u}\right)$, and $L_{u}=0$. This is the lower bound for $L_{u}$.

However, if a unit is maximally segregated, only one group is present in the unit. This means that $p_{g \mid u}=1$ for $g=g^{*}$ (where $g^{*}$ is the group that is present), and $p_{g \mid u}=0$ for $g \neq g^{*}$. In this situation,

$$
E\left(\mathbf{p}_{g \mid u}\right)=-p_{g^{*} \mid u} \log \left(p_{g^{*} \mid u}\right)-\sum_{g \neq g^{*}} p_{g \mid u} \log \left(p_{g \mid u}\right)=0 .
$$

Hence, maximizing the local segregation score is equal to maximizing the cross-entropy:

$$
\begin{aligned}
L_{u} & =E\left(\mathbf{p}_{g \mid u}, \mathbf{p}_{\cdot g}\right)-0 \\
& =-\sum_{g=1}^{G} p_{g \mid u} \log \left(p_{\cdot g}\right) \\
& =-p_{g^{*} \mid u} \log \left(p_{\cdot g^{*}}\right)-\sum_{g \neq g^{*}} p_{g \mid u} \log \left(p_{\cdot g}\right) \\
& =-\log \left(p_{\cdot g^{*}}\right)
\end{aligned}
$$

Clearly, this maximum value will depend on the group distribution, $\mathbf{p} \cdot g$. If we fix $\mathbf{p} \cdot g$, then the maximum value will be

$$
\underset{p \cdot g}{\arg \min }\left(-\log p_{\cdot g}\right) \text {. }
$$

The logic for this is as follows: The most segregated units are those in which only one group is present, and if this group is the smallest in the overall population, the segregation is maximal. This makes intuitive sense. For instance, in a city that is $80 \%$ White and $20 \%$ Black, an all-White neighborhood is segregated, but it is not nearly as segregated as an all-Black neighborhood. This is reflected in the local segregation scores, which are $-\log (0.8)=0.22$ and $-\log (0.2)=1.6$, respectively.

\section{Normalization of local segregation scores}

The previous section shows that, for a fixed group marginal distribution, local segregation scores can range from 0 to $-\log \left(p_{\cdot g^{\prime}}\right)$, where $g^{\prime}$ denotes the smallest group in the population. A possible normalization is therefore to divide local segregation scores by $-\log \left(p_{\cdot g^{\prime}}\right)$, i.e.

$$
L_{u}^{*}=\frac{L_{u}}{-\log \left(p \cdot g^{\prime}\right)} .
$$

Once this is done, the local segregation scores $L_{u}^{*}$ will fall into the range from 0 to 1 . However, it is easy to see that a weighted average of these values will not equal the $H$ index, as

$$
H=\frac{M}{E\left(\mathbf{p}_{\cdot g}\right)} \neq \sum_{u=1}^{U} p_{u} \cdot L_{u}^{*}=\frac{M}{-\log \left(p \cdot g^{\prime}\right)}
$$


The only situation in which there will be equality is when all groups are of equal size in the population, i.e. $p_{\cdot g} \equiv 1 / G$. When this is true, $E\left(\mathbf{p}_{\cdot g}\right)=\log (G)$ and

$$
-\log \left(p_{\cdot g^{\prime}}\right)=-\log (1 / G)=\log (G),
$$

and the above inequality will become an equality. Hence, in situations where the groups under study are of equal size, or approximately of equal size (such as in many studies of occupational segregation), the normalized scores $L_{u}^{*}$ can be useful, as then

$$
H \approx \sum_{u=1}^{U} p_{u} \cdot L_{u}^{*} .
$$

The precision of this approximation can, of course, be checked in empirical studies, and will depend on how good an approximation $-\log \left(p_{\cdot g^{\prime}}\right)$ is for $E\left(\mathbf{p}_{\cdot g}\right)$. In other studies, where the approximation is not good, the $L_{u}^{*}$ can still be useful as they are easier to interpret; however, the researcher should be aware that these scores no longer possess the property that their weighted average forms a recognizable segregation index.

\section{An alternative normalization}

Trivially, local segregation scores could also simply be divided by the entropy of the marginal group distribution, i.e.

$$
L_{u}^{H}=\frac{L_{u}}{E\left(\mathbf{p}_{g}\right)}
$$

It will then be true that

$$
H=\sum_{u=1}^{U} p_{u} \cdot L_{u}^{H} .
$$

However, given that $-\log \left(p_{\cdot g^{\prime}}\right)$ can far exceed $E\left(\mathbf{p}_{\cdot g}\right)$, this is not a normalization in the sense that the $L_{u}^{H}$ are constrained to fall between 0 and 1 . As shown above, in a city that is $80 \%$ White and $20 \%$ Black, the local segregation scores for all-White and all-Black neighborhoods are 0.22 and 1.6, respectively. The entropy of the marginal group distribution is $-0.2 \log (0.2)-$ $0.8 \log (0.8)=0.5$; hence, the "normalized" scores $L_{u}^{H}$ would be 0.44 and 3.2, respectively.

Hence, the $L_{u}^{H}$ can range from 0 to $-\log \left(p_{\cdot g^{\prime}}\right) / E\left(\mathbf{p}_{\cdot g}\right)$. These scores can still be useful when scores that form a weighted average of the form (2) are desired. However, they face the same difficulties of interpretation as the local segregation scores of the $M$ index.

\section{Conclusion}

If we let $g^{\prime}$ denote the smallest group in the population, then the local segregation scores of the $M$ index can range from 0 to $-\log \left(p \cdot g^{\prime}\right)$. A simple normalization of the local segregation scores can hence be achieved by dividing all local segregation scores by $-\log \left(p \cdot g^{\prime}\right)$. These scores will no longer have the useful property that a weighted average of the scores yields the $M$ index. Importantly, these normalized scores will also not aggregate to the $H$ index, unless all groups are of equal size. 
If local segregation scores for the $H$ index are desired, one can divide the local segregation scores of the $M$ index by $E\left(\mathbf{p}_{. g}\right)$, the entropy of the group marginal distribution. However, these scores offer no advantage in terms of interpretation over the local segregation scores of the $M$ index, as they are not constrained to fall into the range from 0 to 1 .

\section{References}

Elbers, Benjamin (2021). "A Method for Studying Difference in Segregation Levels Across Time and Space". In: Sociological Methods \& Research. DOI: 10.1177/0049124121986204.

Mora, Ricardo and Javier Ruiz-Castillo (2011). "Entropy-Based Segregation Indices". In: Sociological Methodology 41.1, pp. 159-194. DOI: 10.1111/j.1467-9531.2011.01237.x.

Reardon, Sean F. and Glenn Firebaugh (2002). "Measures of Multigroup Segregation". In: Sociological Methodology 32.1, pp. 33-67. DOI: 10.1111/1467-9531.00110.

Theil, Henri (1972). Statistical Decomposition Analysis. Amsterdam: North Holland. 